\title{
Pre-Clinical Myocardial Metabolic Alterations in Chronic Kidney Disease
}

\author{
Jeffrey C. Fink ${ }^{a}$ Martin A. Lodge ${ }^{b}$ Mark F. Smith ${ }^{c}$ Anish Hinduja ${ }^{a}$ \\ Jeanine Brown ${ }^{a}$ Mara Y. Dinits-Pensy ${ }^{a}$ Vasken Dilsizian ${ }^{c}$ \\ ${ }^{a}$ Department of Medicine, Division of Nephrology, University of Maryland, ${ }^{b}$ Division of Nuclear Medicine, \\ Russell H. Morgan Department of Radiology and Radiological Sciences, Johns Hopkins University School of \\ Medicine, and ' Department of Diagnostic Radiology and Nuclear Medicine, Division of Nuclear Medicine, \\ University of Maryland, Baltimore, Md., USA
}

For editorial comment see p. 157

\section{Key Words}

Chronic kidney disease $\cdot$ Fluorodeoxyglucose $\cdot$ Glomerular filtration rate $\cdot$ Myocardial metabolism $\cdot$ Positron emission tomography $\cdot$ Uremic cardiomyopathy

\begin{abstract}
The risk for cardiovascular events conferred by decreased renal function is curvilinear with exponentially greater increases in risk as estimated glomerular filtration rate (eGFR) declines. In 13 non-diabetic pre-dialysis chronic kidney disease (CKD) patients, we employed quantitative F-18 fluorodeoxyglucose (FDG) positron emission tomography (PET) as a means to measure myocardial metabolic changes. Methods: Dynamic cardiac FDG PET images were acquired after $6 \mathrm{~h}$ fasting and glucose loading. Corrections for attenuation, scatter, randoms, dead time and decay were applied to the PET data and myocardial glucose utilization (MGU) was calculated using the Patlak method in conjunction with standardized myocardial regions of interest and an image-derived input function (left atrium). MGU was compared with eGFR based on a serum creatinine drawn within 2 weeks of the study date. Results: MGU was relatively uniform between the myocardial sectors (coefficient of variation $=16.2$ $\pm 6.8 \%$ ) within each patient. Between patients, whole myo-
\end{abstract}

\section{KARGER}

(C) 2010 S. Karger AG, Basel

Fax +4161306 1234 E-Mail karger@karger.ch www.karger.com www.karger.com/crd cardium MGU varied considerably with a range of 37.3-156.2 $\mu \mathrm{mol} / \mathrm{min} / 100 \mathrm{~g}$ and a mean of $68.9 \pm 38.3 \mu \mathrm{mol} / \mathrm{min} /$ $100 \mathrm{~g}$. eGFR ranged from $11-89 \mathrm{ml} / \mathrm{min} / 1.73 \mathrm{~m}^{2}$ with a mean of $42.8 \pm 26.9 \mathrm{ml} / \mathrm{min} / 1.73 \mathrm{~m}^{2}$. There was an inverse correlation between whole myocardium MGU and eGFR (Spearman's rho correlation $=-0.615, p=0.025$ ). In multivariate analysis, the relationship between MGU and eGFR was sustained with adjustment for age, race and gender (adjusted $\beta=-1.56 \pm 0.48, p=0.01)$. There was no correlation between cardiac workload and eGFR ( $p=$ NS). Conclusions: A significant inverse correlation between MGU and eGFR is supportive of the hypothesis that CKD is associated with myocardial metabolic changes, which could not be attributed to demographic factors or cardiac workload. Dynamic FDG PET could provide a sensitive, non-invasive, quantitative tool for investigating pre-clinical myocardial abnormalities in patients with CKD.

Copyright $\odot 2010$ S. Karger AG, Basel

\section{Introduction}

Chronic kidney disease (CKD) is recognized as a 'silent epidemic' with more than 19 million Americans affected by the disorder [1]. Cardiovascular disease is the 
Fig. 1. Sequence and timing of FDG PET acquisition protocol.

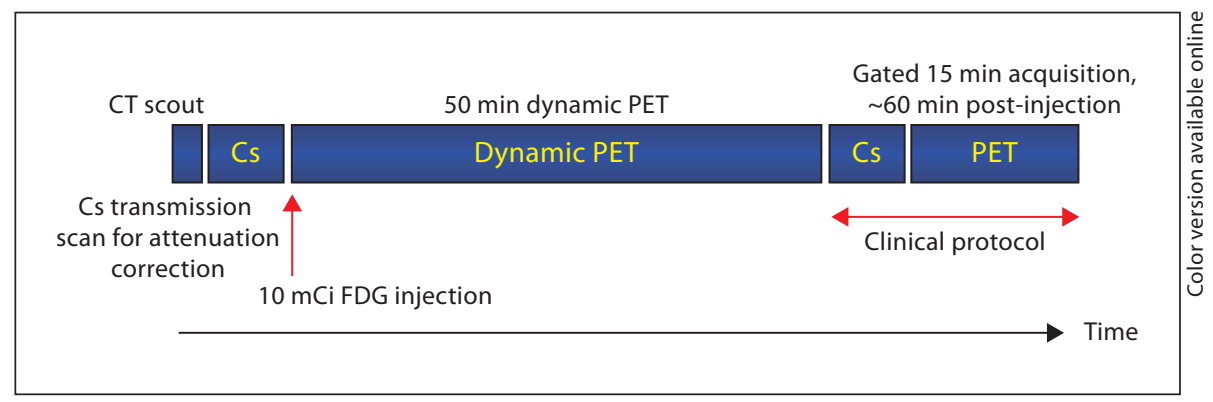

primary cause of the morbidity and mortality observed with all forms of kidney disease at any stage, which occurs at a disproportionate rate in patients with pre-dialysis CKD [1-5]. The risk for cardiovascular events conferred by decreased renal function increases as estimated glomerular filtration rate (eGFR) declines, with a key inflection point at eGFR values of $45 \mathrm{ml} / \mathrm{min} / 1.73 \mathrm{~m}^{2}$ and less [5].

Individuals with CKD are faced with both pressureand volume-overload states contributing to the development of maladaptive left ventricular remodeling and heart failure. The heart failure observed in CKD may include both systolic and diastolic dysfunction, but also has unique changes associated with uremia [6-8]. The cardiomyopathy of uremia is thought to lead to myocyte-tocapillary mismatch, with a diminished vascular supply relative to the number and volume of functioning myocytes [9-11]. The oxygen-poor milieu may lead to diffuse myocardial ischemia with an anticipated decline in aerobic myocardial fatty acid utilization and a shift to anaerobic glucose utilization [12-14]. The shift from a predominance of aerobic (fatty acid) to anaerobic (glucose) metabolism may account for a significant portion of the excessive cardiovascular morbidity and mortality observed in kidney disease [15]. This pathologic transformation that eventually promotes myocardial fibrosis is most evident in end-stage renal disease and is associated with excessive mortality; however, it is likely to develop in early stages of impaired renal function [16-18].

In the current study, we hypothesized that in patients with pre-dialysis CKD, myocyte-to-capillary mismatch is a progressive disorder, which accrues with loss of renal function, and is associated with a shift in cardiac energetics from a predominance of aerobic to anaerobic metabolism, where glucose is the primary fuel. Because the distribution of myocardial metabolism in the left ventricle can appear homogeneous and visually normal, when in fact it may be diffusely abnormal in absolute terms, we proposed to assess the feasibility of measuring myocardial glucose utilization (MGU) quantified, in $\mu \mathrm{mol} /$ min/100 g, using the Patlak method [19]. The primary outcome variable of interest, MGU, was considered as a continuous variable and we assessed its reliability of measurement in the disease population. As a secondary objective, we examined the relationship between MGU and renal function as measured by eGFR.

\section{Methods}

This was a pilot cross-sectional study to evaluate the feasibility of using F-18 fluorodeoxyglucose (FDG) positron emission tomography (PET) to measure myocardial metabolism in pre-dialysis CKD patients. Study participants with stage II-V CKD, related to a variety of proteinuric and non-diabetic kidney diseases, were selected with a range in renal function as indicated by eGFR, which was based on a serum creatinine level determined within 2 weeks of the study date. The eGFR was calculated with the abbreviated Modified Diet in Renal Disease equation, which has been accepted as a non-invasive alternative to direct measurement of GFR [20]. Potential study participants were excluded if they had a history of diabetes mellitus or overt cardiovascular disease including angina, myocardial infarction, arrhythmia or congestive heart failure or an EKG demonstrating ischemic heart disease. However, patients with hypertension or left ventricular hypertrophy were not excluded. Study participants provided written consent to participate in this institutional review board-approved study.

On the day of study, each participant received an oral carbohydrate-rich solution (50 g Glucola $\left.{ }^{\circledR}\right)$ after a six-hour fast consistent with established protocols [21]. Plasma glucose was monitored by finger-stick and when measured to be under $200 \mathrm{mg} / \mathrm{dl}$ at one hour or later, $370 \mathrm{MBq}(10 \mathrm{mCi})$ of FDG was intravenously injected. The time interval from the administration of the Glucola to the time of FDG injection was $60 \mathrm{~min}$. In study participants with glucose readings greater than or equal to $200 \mathrm{mg} / \mathrm{dl}$, insulin was given to achieve target glucose prior to FDG administration. Images were obtained using a Gemini PET/CT scanner (Philips Medical Systems, The Netherlands). The PET acquisition protocol is shown in figure 1 . Thirty-five dynamic frames $(20 \times 15 \mathrm{~s}$ and $15 \times 180 \mathrm{~s}$ ) were obtained over $50 \mathrm{~min}$ starting immediately 


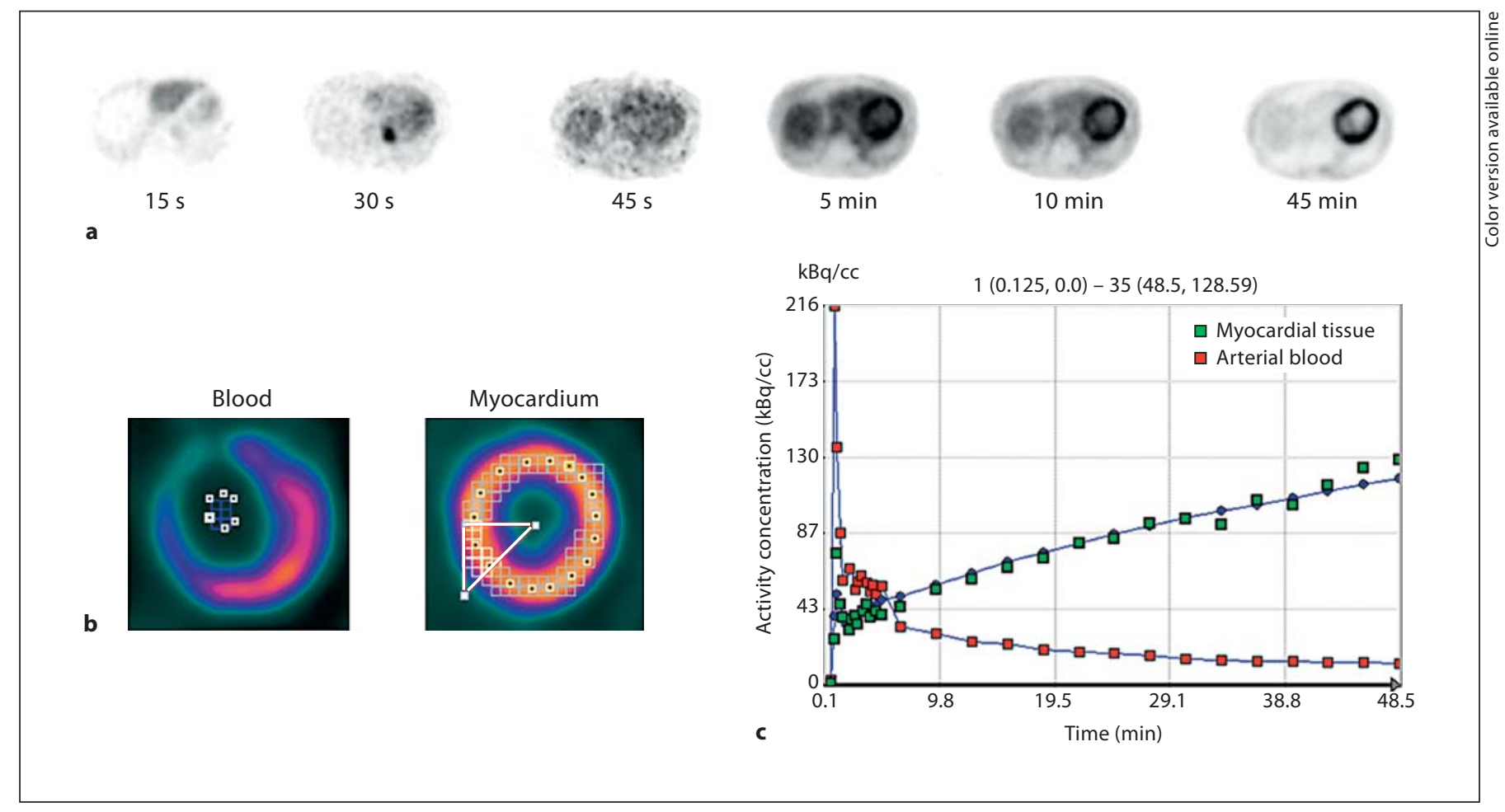

Fig. 2. a Individual images, illustrating the path of FDG bolus through the cardiac blood pool into the left ventricular myocardium over a span of $45 \mathrm{~min}$. b Region of interest overlay over short axis left ventricular myocardium and blood pool slices. c Time-activity curves. Dynamic imaging of the heart allows analysis of the FDG concentration in both arterial blood and myocardial tissue as a function of time. Green squares (light grey in the print version) represent the activity concentration in myocardial tissue and the red squares (dark grey in the print version) represent the activity concentration in arterial blood.

after FDG injection. Transverse images were reconstructed to give a spatial resolution of approximately $8-\mathrm{mm}$ full width at half maximum. Images were corrected for randoms (online subtraction of delayed events), dead time (singles-based correction), scatter (single scatter simulation), attenuation $\left({ }^{137} \mathrm{Cs}\right.$ transmission source), and radioactive decay. Gated FDG PET images were acquired to assess left ventricular function, mass and volumes in 12 of 13 subjects.

The dynamic images were analyzed using the PMOD biomedical image quantification software (PMOD Technologies, Switzerland). Transverse images were first re-sliced to the short axis orientation and region of interests (ROIs) were defined on all myocardial slices using the last frame of image data. These regions were semi-automatically grouped to form 16 ROIs covering the standard cardiac segments and an additional whole-myocardium ROI was defined. The 16 myocardial ROIs were manually defined by drawing an irregular line around the center of the myocardial wall in all slices. The ROIs were determined from this line by considering pixels that lay within a $15-\mathrm{mm}$-thick region around this line. ROIs were then grouped into the standard myocardial sectors. Input functions were estimated from additional ROIs manually defined in the left atrium. The blood pool ROI in the left atrium was $8-12 \mathrm{~mm}$ across, defined in 4 - or 5-image slices. ROI analysis of the dynamic image data produced timeactivity curves for blood and the different sectors of the myocardium (fig. 2).

Cardiac workload in joules/min was computed by the product of cardiac output and mean arterial pressure. Cardiac output was calculated from the product of heart rate and stroke volume, which was derived from the gated FDG PET images. Cardiac work computed in units of $1 \cdot \mathrm{mm} \mathrm{Hg} / \mathrm{min}$ was converted to joules/min by multiplying with 0.1334 [22]. Cardiac work was also normalized to left ventricular myocardial mass (joules/g/min) obtained from gated PET images.

Following i.v. injection, FDG is rapidly exchanged across the capillary and cellular membranes, phosphorylated by hexokinase to FDG-6-phosphate, and is not metabolized further nor used in glycogen synthesis. Thus, FDG-6-phosphate was assumed to be irreversibly bound within the period of scanning. Tracers that are irreversibly bound can be analyzed using the Patlak approach. Patlak analysis was used to determine MGU of all ROIs [19]. The plasma glucose concentration for the Patlak graphical analysis was obtained immediately before the FDG injection. The lumped constant, which accounts for differences in the affinities of transmembrane transport and phosphorylation of FDG and the glucose molecule, was set to a value of 0.67 . 

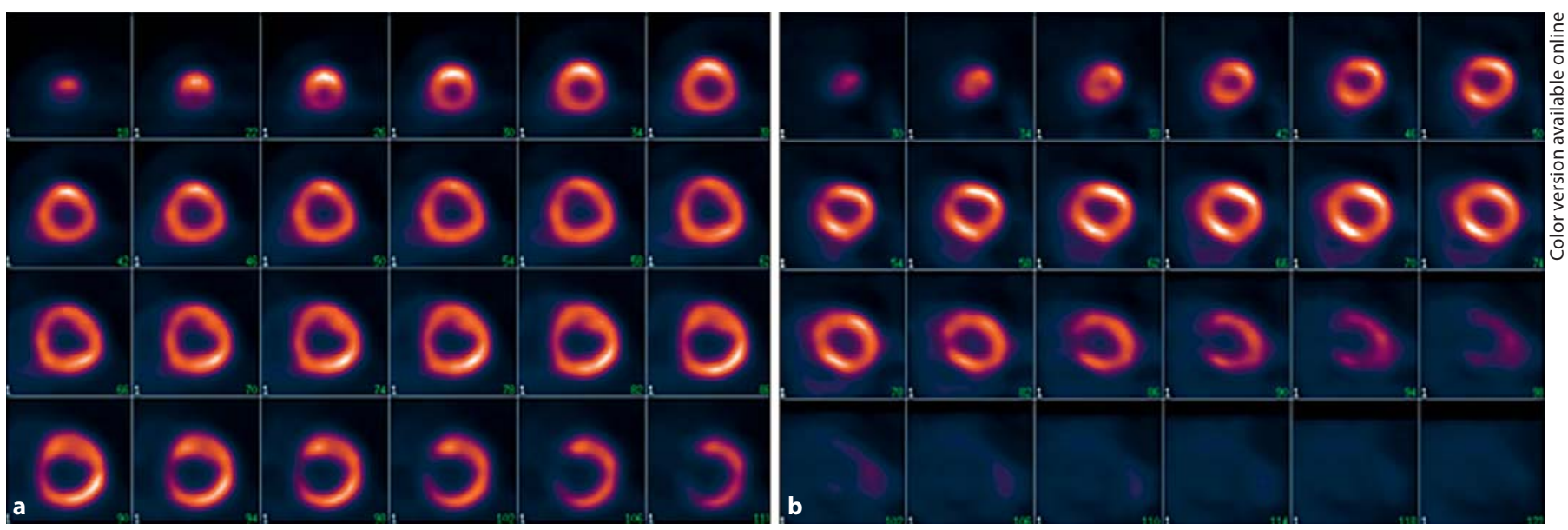

Fig. 3. Examples of myocardial FDG distribution in CKD patients exhibiting no visually discernable regional defects. a Representative example of a CKD patient with dilated left ventricular cavity and concentric hypertrophy. b CKD patient with normal left ventricular cavity size without hypertrophy.

All continuous parameters are reported with means \pm SD and categorical variables were reported with $\mathrm{n}(\%)$. We employed the coefficient of variation, using the quotient of the SD of the 16 ROIs and the mean of ROIs, to assess variability of PET measurements within subjects. The non-parametric Spearman's correlation coefficient was employed to examine the relationship between MGU and eGFR. A p value of $\leq 0.05$ was considered statistically significant.

\section{Results}

The demographic characteristics of the 13 patients with stage II-V CKD are shown in table 1 . Two study subjects required 1-2 units of regular insulin as a consequence of unexpected glucose intolerance following the Glucola load.

Consistent with the exclusion criteria of patients with known coronary artery disease, all study participants had physiologic myocardial FDG distributions without visually discernable regional defects (fig. 3). Similarly, quantitative MGU was relatively uniform between the myocardial sectors (coefficient of variation $=16.2 \pm 6.8 \%$ ), which supports the notion that there is minimal heterogeneity in the regional uptake among CKD patients who were screened using a clinical criteria to exclude ischemic heart disease. In contrast, whole myocardium MGU varied considerably between CKD subjects, with a range of $37.3-156.2 \mu \mathrm{mol} / \mathrm{min} / 100 \mathrm{~g}$ and a mean of $68.9 \pm 38.3$ $\mu \mathrm{mol} / \mathrm{min} / 100 \mathrm{~g}$. The variation across the subjects was much wider than the average variation within subjects
Table 1. Characteristics of study participants $(n=13)$

\begin{tabular}{lc}
\hline Characteristic & \\
\hline Age, years & $45.6 \pm 10.2$ \\
Sex & \\
$\quad$ Male & $9(69.2)$ \\
$\quad$ Female & $4(30.8)$ \\
Race & \\
$\quad$ African-American & $12(92.3)$ \\
$\quad$ Caucasian & $1(7.7)$ \\
Weight, kg & $86.7 \pm 21.1$ \\
Mean arterial pressure, mm Hg & $105.2 \pm 12.0$ \\
Serum creatinine, mg/dl & $2.9 \pm 1.9$ \\
eGFR, ml/min/1.73 m ${ }^{2}$ & $42.8 \pm 26.9$ \\
Baseline electrocardiogram with criteria & \\
for left ventricular hypertrophy & \\
$\quad$ Yes & $2(15.4)$ \\
$\quad$ No & $11(83.3)$ \\
Non-specific ST-T wave abnormality & \\
$\quad$ Yes & $4(30.8)$ \\
$\quad$ No & $9(69.2)$ \\
Fasting blood glucose, mg/dl & \\
$\quad$ Baseline & \\
1 h after Glucola administration & $170.5 \pm 50.7$ \\
Received insulin after Glucola & \\
$\quad$ Yes & $2(15.4)$ \\
$\quad$ No & $11(83.33)$ \\
&
\end{tabular}

Numbers in parentheses are percentages. 


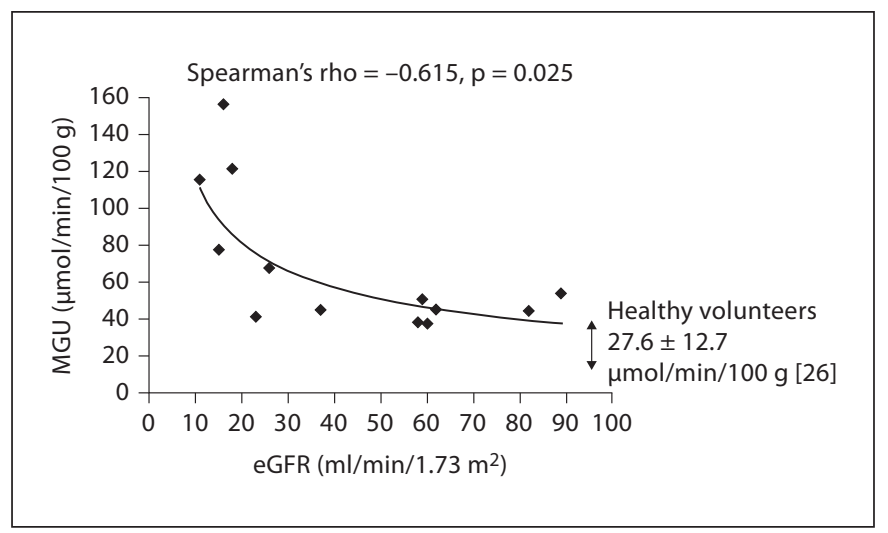

Fig. 4. Scatter plot of eGFR versus $\mathrm{MGU}$ as measured by ${ }^{18} \mathrm{FDG}$ PET scan in CKD. The fitted line is $\mathrm{MGU}=\mathrm{k} \cdot \mathrm{eGFR}^{\mathrm{x}}$ with estimated fitting parameters $\mathrm{k}=385.2(\mathrm{p}<0.00001)$ and $\mathrm{x}=-0.520$ $(\mathrm{p}=0.002)$.

across their respective ROIs, and the inter-subject variability is partially explained by the relationship between MGU and eGFR (fig. 4).

eGFR values ranged from 11 to $89 \mathrm{ml} / \mathrm{min} / 1.73 \mathrm{~m}^{2}$, with a mean of $42.8 \pm 26.9 \mathrm{ml} / \mathrm{min} / 1.73 \mathrm{~m}^{2}$. There was an inverse correlation between whole myocardium MGU and eGFR (Spearman's rho value $=-0.615, \mathrm{p}=0.025$; fig. 4). In multivariate models with MGU as the response variable and eGFR with age, race, and gender as key covariates, we found the three demographic characteristics taken together to be significant predictors of MGU, accounting for a substantial proportion of its variance $\left(\mathrm{R}^{2}=0.32\right)$. When eGFR was also included in the multivariate analysis, the modeled variance more than doubled $\left(\mathrm{R}^{2}=0.70\right)$, with eGFR accounting for a greater part of the overall variance $(38 \%)$ than the demographic variables (32\%). From this latter multivariate model, eGFR was a significant predictor of MGU (regression coefficient $\beta=$ $-1.56 \pm 0.48, \mathrm{p}=0.01$ ). The fitted line in figure 4 is of the form $\mathrm{MGU}=\mathrm{k} \cdot \mathrm{eGFR}^{\mathrm{x}}$. The parameters $\mathrm{k}$ and $\mathrm{x}$ were found by linear least squares fitting of the transformed equation $\ln (\mathrm{MGU})=\ln (\mathrm{k})+\mathrm{x} \cdot \ln (\mathrm{eGFR})$ for $\ln (\mathrm{k})$ and $\mathrm{x}$. The estimated values were $\mathrm{k}=385.2(\mathrm{p}<0.00001)$ and $\mathrm{x}=-0.520(\mathrm{p}=0.002)$. The $95 \%$ confidence intervals for $\mathrm{k}$ and $\mathrm{x}$ were 137 to 1,079 and -0.806 to -0.233 , respectively.

To determine whether cardiac work could explain the detected relationship between MGU and eGFR, the workload of the heart was estimated from blood pressure and stroke volume in units of $1 \cdot \mathrm{mm} \mathrm{Hg} / \mathrm{min}$ and converted to joules/min [21]. There was no correlation between cardiac work and eGFR ( $r=0.037, p=N S$; fig. 5). Similarly, when the workload of the heart was normalized to left
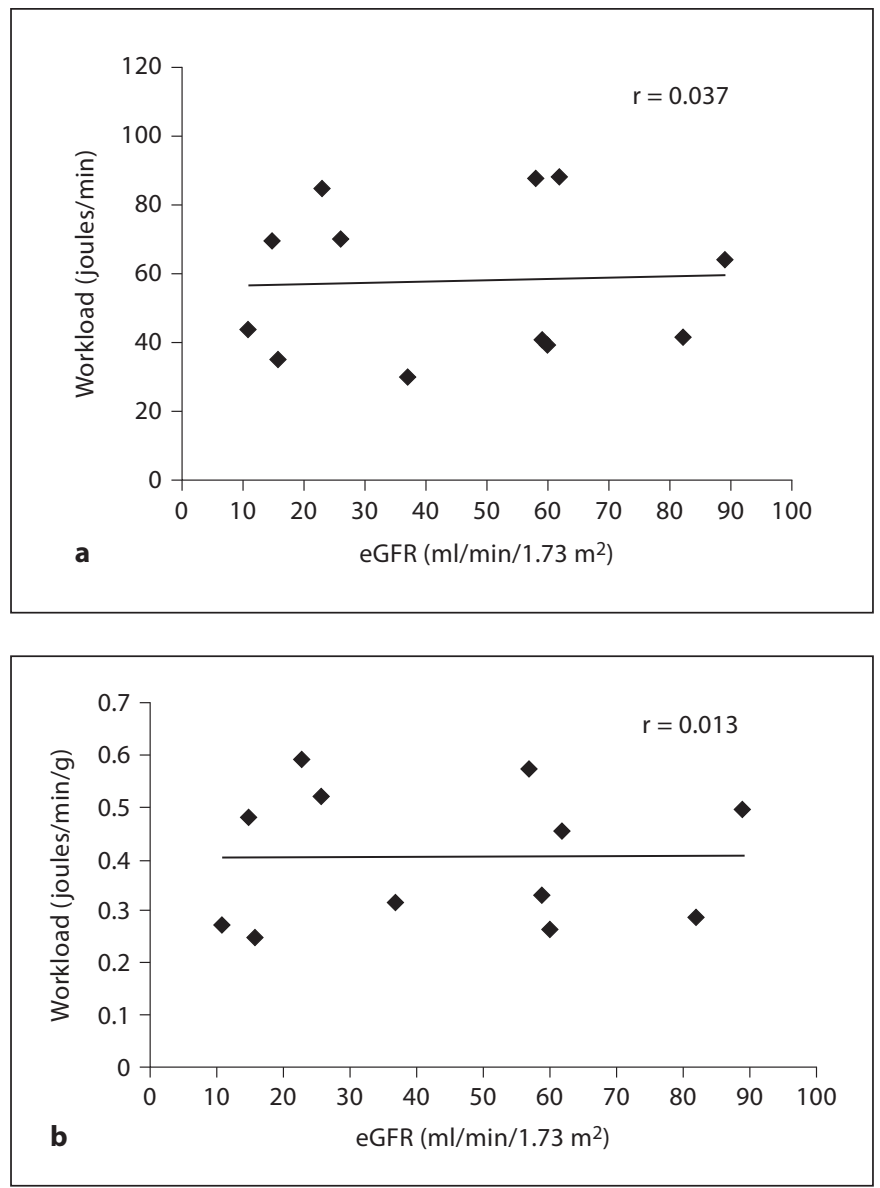

Fig. 5. Scatter plot of eGFR $\left(\mathrm{ml} / \mathrm{min} / 1.73 \mathrm{~m}^{2}\right)$ versus cardiac work (a) and cardiac work normalized to left ventricular myocardial mass (b) as measured by gated ${ }^{18}$ FDG PET scan in CKD. The data represent 12 of 13 subjects with gated PET images.

ventricular myocardial mass (joules/g/min), there was no relationship with eGFR $(r=0.013, p=N S)$. The lack of link between workload and eGFR persisted when systolic blood pressure instead of mean arterial blood pressure was used to compute cardiac work.

\section{Discussion}

CKD is a major public health problem associated with excessive cardiovascular morbidity and mortality, high health care costs, and impaired quality of life [5, 22-25]. Thus, the application of a non-invasive, quantitative PET technique to examine myocardial metabolism and gain insight into the deleterious effect of altered myocardial metabolism in kidney disease has clinical relevance. 
The current study confirms the feasibility of employing quantitative MGU with PET to gain insight into the alterations of myocardial metabolism in CKD patients, which might not be detected by visual inspection alone. Along with the minimal intra-individual variability of the MGU measurements, which can be used as a measure of precision, we also demonstrated a significant relationship between myocardial metabolism, assessed by MGU, and renal function, assessed by eGFR, in CKD patients. There was no link between cardiac work and eGFR to explain the detected relationship between MGU and eGFR. The pilot data revealed that as GFR decreased across individuals, there was a rise in MGU across the study participants. The relatively minimal coefficients of variation within individuals supported the use of the clinical screening method as an effective means of excluding patients with significant coronary artery disease, which might lead to heterogenous FDG uptake.

Of great interest is the degree to which MGU increased with declining renal function across individuals in the study sample even when adjusting for differences in age, race and gender. This was particularly notable among the sub-group with significant impairment in renal function, with stage III or greater CKD. This correlation was substantial despite the small number of study participants. The significant $p$ value for this correlation indicates that the likelihood of this relationship occurring by chance, even with a small number of study participants, is quite low. Moreover, the range in values observed for MGU was on a comparable scale, but exceeded the values reported for healthy persons $(27.6 \pm 12.7 \mu \mathrm{mol} / \mathrm{min} / 100 \mathrm{~g})$ [26] and in patients with heart failure with and without diabetes and coronary disease $(11-12.3 \mu \mathrm{mol} / \mathrm{min} / 100 \mathrm{~g})$ $[27,28]$.

The utility of FDG PET for the assessment of myocardial metabolism in patients with chronic left ventricular dysfunction is well established [29]. FDG PET images are usually interpreted qualitatively in areas with myocardial perfusion deficits at rest and designated as either metabolism-perfusion mismatch (indicating viable myocardium) or match (indicating scarred myocardium) defects. It is important to point out, however, that these parameters of mismatch and match represent end stages of cardiovascular disease with occlusive coronary artery disease. Detection of early, pre-clinical myocardial metabolic alterations can be limited with qualitative assessment of regional FDG uptake. Although the distribution of FDG uptake may appear homogeneous throughout the left ventricular myocardium, absolute MGU may be abnormal. Quantitative assessment with PET may identify alterations in MGU in the heart before clinical, function$\mathrm{al}$, and prognostic consequences ensue [30]. Normal cardiac metabolism is comprised predominately (approximately 70\%) of aerobic metabolism, with free fatty acids used as the energy substrate. Under conditions of stress, cardiac metabolism shifts to anaerobic metabolism, where glucose serves as the principal fuel. The price of this switch in metabolism is a less efficient ATP production, even though less oxygen is required for its synthesis. Investigators have used single-photon emission computed tomography with the fatty acid analogue iodine-123$\beta$-methyl iodophenyl-pentadecanoic acid to demonstrate alterations in myocardial fatty acid utilization in endstage renal disease patients (without coronary artery disease), which was strongly correlated with systemic glucose intolerance and systolic left ventricular dysfunction [31]. In another study, impaired myocardial fatty acid metabolism in asymptomatic patients with end-stage renal disease was able to identify the subgroup of patients who were at high risk for cardiac death [15]. The shift from a predominance of aerobic (fatty acid) to anaerobic (glucose) metabolism appeared to account for a significant portion of the excessive cardiovascular morbidity and mortality observed across all stages of kidney disease. These findings support the assertion that altered cardiac metabolism (indicating silent myocardial ischemia) is highly prevalent in patients with renal disease and can identify the subgroup of patients who are at high risk for cardiac death.

There may be alternative explanations for the significant rise in MGU seen in the CKD population. For example, does uremia per se increase MGU? In isolated perfused rat hearts, abnormal carbohydrate metabolism was demonstrated in 'acute' uremic rat hearts, with impairment of glucose transport, hexokinase activity, and abnormal glycogen metabolism, but not in 'chronic' uremic animals [32]. When compared to chronic sham-operated animals, chronic uremic animals had less cardiac glycogen content, but there were no differences in glucose or glycogen metabolism or in response to insulin. What about age? The CKD population, with its high degree of co-morbidity, may be subject to complications seen with aging. Myocardial fatty acid metabolism tends to decrease with aging, possibly due to a decrease in carnitine palmitoyltransferase- 1 activity, which is the rate-limiting step in fatty acid metabolism [33]. Alternatively, there may be an increase in oxygen free radical injury leading to mitochondrial dysfunction, which impedes fatty acid metabolism [33]. Additionally, with a high preponderance of insulin resistance in $\mathrm{CKD}$, there may be an inhi- 
bition of the fatty acid metabolism as a result of hyperinsulinemia [34]. Finally, one must ask whether the alterations in myocardial metabolism observed among patients with CKD in this study are a consequence, rather than a cause, of the development of heart failure in this disease population, since fatty acid metabolism is also decreased in patients with dilated cardiomyopathy [35]. In future studies, measures of serum insulin, free fatty acid, and lactate levels may provide additional insight into alternative mechanistic explanations for the significant rise in MGU seen in the CKD population.

In conclusion, among non-diabetic CKD patients without overt coronary artery disease, there was a significant inverse correlation between MGU (measured in $\mu \mathrm{mol} / \mathrm{min} / 100 \mathrm{~g}$ of myocardial tissue) and eGFR, which could not be attributed to differences in demographic characteristics or cardiac workload. In the future, dynamic FDG PET may provide a sensitive, non-invasive, quantitative tool for investigating pre-clinical myocardial abnormalities in patients with CKD.

\section{Acknowledgment}

This project was supported by grant 1P60 MD000532-01 from the National Center on Minority Health and Health Disparities. Its contents are solely the responsibility of the authors and do not necessarily represent the official views of the NCMHD.

\section{References}

1 Coresh J, Selvin E, Stevens LA, Manzi J, Kusek JW, Eggers P, Van Lente F, Levey AS: Prevalence of chronic kidney disease in the United States. JAMA 2007;298:2038-2047.

-2 Mann JF, Gerstein HC, Pogue J, Bosch J, Yusuf S: Renal insufficiency as a predictor of cardiovascular outcomes and the impact of ramipril: the HOPE randomized trial. Ann Intern Med 2001;134:629-636.

-3 Meier-Kriesche HU, Baliga R, Kaplan B: Decreased renal function is a strong risk factor for cardiovascular death after renal transplantation. Transplantation 2003;75:12911295.

-4 Weiner DE, Tighiouart H, Amin MG, Stark PC, MacLeod B, Griffith JL, Salem DN, Levey AS, Sarnak MJ: Chronic kidney disease as a risk factor for cardiovascular disease and all cause mortality: a pooled analysis of community based studies. J Am Soc Nephrol 2004;15:1307-1315.

5 Go AS, Chertow GM, Fan D, McCulloch CE, Hsu CY: Chronic kidney disease and the risks of death, cardiovascular events, and hospitalization. N Engl J Med 2004;351: 1296-1305.

-6 Foley RN, Parfrey PS, Harnett JD, Kent GM, Martin CJ, Murray DC, Barre PE: Clinical and echocardiographic disease in patients starting end-stage renal disease therapy. Kidney Int 1995;47:186-192.

-7 Hayashi SY, Rohani M, Lindholm B, Brodin LA, Lind B, Barany P, Alvestrand A, Seeberger A: Left ventricular function in patients with chronic kidney disease evaluated by colour tissue Doppler velocity imaging. Nephrol Dial Transplant 2006;21:125-132.

$\checkmark 8$ Ahmed A, Rich MW, Sanders PW, Perry GJ, Bakris GL, Zile MR, Love TE, Aban IB, Shlipak MG: Chronic kidney disease associated mortality in diastolic versus systolic heart failure: a propensity matched study. Am J Cardiol 2007;99:393-398.
9 Mall G, Huther W, Schneider J, Lundin P, Ritz E: Diffuse intermyocardiocytic fibrosis in uraemic patients. Nephrol Dial Transplant 1990;5:39-44.

10 Amann K, Wiest G, Zimmer G, Gretz N, Ritz E, Mall G: Reduced capillary density in the myocardium of uremic rats: a stereological study. Kidney Int 1992;42:1079-1085.

-11 Amann K, Breitbach M, Ritz E, Mall G: Myocyte/capillary mismatch in the heart of uremic patients. J Am Soc Nephrol 1998;9:10181022.

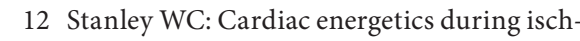
emia and the rationale for metabolic interventions. Coron Artery Dis 2001;12:S3-S7.

13 van der Vusse GJ, Stam H: Lipid and carbohydrate metabolism in the ischemic heart Basic Res Cardiol 1987;82:149-153.

14 Tyralla K, Amann K: Morphology of the heart and arteries in renal failure. Kidney Int 2003;63:S80-S83.

15 Nishimura M, Tsukamoto K, Hasebe N, Tamaki N, Kikuchi K, Ono T: Prediction of cardiac death in hemodialysis patients by myocardial fatty acid imaging. J Am Coll Cardiol 2008;51:139-145.

16 Aoki J, Ikari Y, Nakajima H, Mori M, Sugimoto T, Hatori M, Tanimoto S, Amiya E, Hara K: Clinical and pathologic characteristics of dilated cardiomyopathy in hemodialysis patients. Kidney Int 2005;67:333-340.

-17 Mark PB, Johnston N, Goenning BA, Foster JE, Blyth KG, Martin TN, Steedman T, Dargie HJ, Jardine AG: Redefinition of uremic cardiomyopathy by contrast enhanced cardiac magnetic resonance imaging. Kidney Int 2006;69:1839-1845.

18 Pizzarelli F, Dattolo P, Ferdeghini EM, Morales MA: Parameters derived by ultrasonic myocardial characterization in dialysis patients are associated with mortality. Kidney Int 2005;68:1320-1325.
19 Patlak CS, Blasberg RG, Fenstermacher JD: Graphical evaluation of blood-to-brain transfer constants from multiple-time uptake data. J Cereb Blood Flow Metab 1983;3: $1-7$.

20 Levey AS, Stevens LA, Schmid CH, Zhang YL, Castro AF 3rd, Feldman HI, Kusek JW, Eggers P, Van Lente F, Greene T, Coresh J: A new equation to estimate glomerular filtration rate. Ann Intern Med 2009;150:604612.

21 Laine H, Katoh C, Luotolahti M, Yki-Järvinen H, Kantola I, Jula A, Takala TO, Ruotsalainen U, Iida $H$, Haaparanta M, Nuutila P, Knuuti J: Myocardial oxygen consumption is unchanged but efficiency is reduced in patients with essential hypertension and left ventricular hypertrophy. Circulation 1999; 100:2425-2430.

22 Schiffrin EL, Lipman ML, Mann JF: Chronic kidney disease: effects on the cardiovascular system. Circulation 2007;116:85-97.

23 National Kidney Foundation: K/DOQI clinical practice guidelines for chronic kidney disease: evaluation, classification, and stratification. Am J Kidney Dis 2002;39:S1-S266.

24 Sarnak MJ, Levey AS, Schoolwerth AC, Coresh J, Culleton B, Hamm LL, McCullough PA, Kasiske BL, Kelepouris E, Klag MJ, Parfrey P, Pfeffer M, Raij L, Spinosa DJ, Wilson PW: Kidney disease as a risk factor for development of cardiovascular disease: a statement from the American Heart Association Councils on Kidney in Cardiovascular Disease, High Blood Pressure Research, Clinical Cardiology, and Epidemiology and Prevention. Circulation 2003; 108:21542169.

25 Tonelli M, Wiebe N, Culleton B, House A, Rabbat C, Fok M, McAlister F, Garg AX: Chronic kidney disease and mortality risk: a systematic review. J Am Soc Nephrol 2006; 17:2034-2047. 
-26 Morita K, Katoh C, Yoshinaga K, Noriyasu K, Mabuchi M, Tsukamoto T, Kageyama H, Shiga T, Kuge Y, Tamaki N: Quantitative analysis of myocardial glucose utilization in patients with left ventricular dysfunction by means of ${ }^{18} \mathrm{~F}$-FDG dynamic positron tomography and three-compartment analysis. Eur J Nucl Med Mol Imaging 2005;32:806-812.

-27 Taylor M, Wallhaus TR, Degrado TR, Russell DC, Stanko P, Nickles RJ, Stone CK: An evaluation of myocardial fatty acid and glucose uptake using PET with [18F]fluoro-6-thiaheptadecanoic acid and [18F]FDG in patients with congestive heart failure. J Nucl Med 2001;42:55-62.
28 Vitale GD, deKemp RA, Ruddy TD, Williams K, Beanlands RS: Myocardial glucose utilization and optimization of [18]F-FDG PET imaging in patients with non-insulindependent diabetes mellitus, coronary artery disease, and left ventricular dysfunction. J Nucl Med 2001;42:1730-1736.

29 Tillisch JH, Brunken R, Marshall R, Schwaiger M, Mandelkern M, Phelps M, Schelbert H: Reversibility of cardiac wallmotion abnormalities predicted by positron tomography. N Engl J Med 1986;314:884888.

30 Dilsizian V, Fink J: Deleterious effect of altered myocardial fatty acid metabolism in kidney disease. J Am Coll Cardiol 2008;51 146-148.

-31 Nishimura M, Murase M, Hashimoto T, Kobayashi H, Yamazaki S, Imai R, Okino K, Fujita $\mathrm{H}$, Inoue $\mathrm{N}$, Takahashi $\mathrm{H}$, Ono T: Insulin resistance and impaired myocardial fatty acid metabolism in dialysis patients with normal coronary arteries. Kidney Int 2006; 69:553-559.
2 Penpargkul S, Kuziak J, Scheuer J: Effect of uremia upon carbohydrate metabolism in isolated perfused rat heart. J Mol Cell Cardiol 1975;7:499-511.

33 Kates AM, Herrero P, Dence C, Soto P, Srinivasan M, Delano DG, Ehsani A, Gropler RJ: Impact of aging on substrate metabolism by the human heart. J Am Coll Cardiol 2003;41: 293-299.

34 Neely JR, Morgan HE: Relationship between carbohydrate metabolism and energy balance of heart muscle. Ann Rev Physiol 1974; 36:413-459.

35 Dávila-Román VG, Vedala G, Herrero P, de las Fuentes L, Rogers JG, Kelly DP, Gropler $\mathrm{RJ}$ : Altered myocardial fatty acid and glucose metabolism in idiopathic dilated cardiomyopathy. J Am Coll Cardiol 2002;40:271-277. 